Session 1520

\title{
Trial Results from a Personalized Wireless On-Site Reference Distance Learning Solution
}

\author{
Carlos R. Morales, Jason Sole \\ Purdue University
}

\begin{abstract}
This paper provides results from an implementation trial of a mobile, wireless, streamingvideo instructional-delivery system developed by the authors. Specifically, the authors present their findings from their experiment to determine the effectiveness of using wireless PDA's for on-site instructional delivery. The authors have subjects attempt to perform a procedural task that is foreign to them. They measure the time and number of mistakes made by the subjects in five different scenarios.
\end{abstract}

From a technical perspective, the authors employ a system capable of delivering fullscreen full-motion streaming video, and hyperlinked context sensitive illustrations and schematics to learners over an 11- megabit IEEE 802.11 wireless network.

\section{Introduction}

The pervasiveness of Personal Digital Assistants [PDAs] and wireless technologies has created a new form of instructional delivery. Until recently the delivery of instructional materials to computing devices required the use of full-blown computers or laptops. Thus, most distance learning occurred within the confines of rooms for that purpose. This has changed.

A new term has emerged to describe the delivery of training to mobile devices such as PDA or WAP enabled phones. M-Learning or Mobile-Learning attempts to bring knowledge to users on-site at their primary activity or environment. Companies such as InterWise, GlobalKnowledge, and KnowledgePlanet have made it a priority to address this new market. "All of these companies see huge opportunity for this type of delivery, as millions of mobile professionals worldwide use handheld wireless devices, with mullions more expected to join their ranks in the coming years." [1]

This proliferation can be partially attributed to a few factors. First, it is relative inexpensive to set-up a system capable of delivering training on-site. Morales details a system than can be assembled for less than one thousand dollars [2]. Second, the types of skills to set-up a delivery system of this type are relatively easy to obtain. With a basic knowledge of server-side web-development and networking any organization can set-up an m-learning system.

Intuitively, it would seem that one could create an extremely efficient learning system by focusing on the delivery of instructional-materials to users while they are engaged in their primary environment. This would contribute to efficiency in three ways. First, learners would not have to be pulled off-line while being trained. They would be asked to perform tasks and provided with the requisite knowledge in a "just-in-time" manner [3]. This would eliminate the down-time often associated with training. Second, there would

"Proceedings of the 2002 American Society for Engineering Education Annual Conference \& Exposition Copyright (C) 2002, American Society for Engineering Education", 
a smaller chance for the learners to forget the knowledge required to performed their task. Finally, the use of strong visuals such as pictures, diagrams and movies would minimize mistakes resulting from misidentification of tools or parts. This was the argument presented by Morales at an Information Systems Division session during the 2001 ASEE National Meeting [2].

In this paper we test the strength of using PDA for instructional delivery. To accomplish this task, the authors created an experiment in which subjects were asked to perform a procedural task that was foreign to them. In order to isolate the, the authors conducted five different treatments and tracked the amount of time and mistakes made by the subjects.

\section{Experimental Procedures}

\section{Overview}

The subjects were asked to assemble a flashing LED using an electronic kit from Radio Shack. None of the subjects has experience performing this type of task. The authors measured the number of mistakes and the amount of time required for subjects to complete the task. A total of give groups and treatments were used to isolate the effects of the PDA as an on-site reference.

\section{Experiment Design:}

The experiment was designed to test several scenarios in order to determine which combination of instructions and electronic assistance generate the best results in the assembly of the test object.

\section{The Test Object:}

The test object was a simple transistor board purchased from Radio Shack for $\$ 49.00$. It consisted of eleven steps and included schematics and instruction printed on paper that indicated the proper way to assemble the board.

\section{The Test Subjects:}

The test subjects were students drawn from a pool of three classes in the Computer Graphics Department at Purdue University. The classes all centered around multimedia development, and between the three of them covered the creation of digital video, server side scripting, and Lingo scripting. The students in the three classes were pooled, and then assigned a number according to alphabetical order. Then using software, twentynine numbers were chosen at random. The students with corresponding numbers were then chosen as possible candidates. The students were then approached and asked if they would be willing to participate in the study, which we estimated would take no more than an hour of their time. Of the twenty-nine, twenty-seven agreed. Of those, two had prior knowledge of the transistor board assembly, and were therefore disqualified from the sample. Of the remaining twenty-five, the first twenty who volunteered were selected for the study. 


\section{Small Sample Size:}

The authors elected to use a relatively small sample size for this experiment to establish determine the need for a large-scale study and to minimize contamination due to equipment shortage. It took approximately seven hours to test each subject, because the equipment only allowed for a single subject to be tested at a time. The authors were concerned that inter-subject contamination would be greater if the trials went on for more than a single day. Thus, authors elected to use as many subjects as they could tests in a single 8-hour day with a single set of equipment.

Based on the results of this study, the authors have been able to justify the necessary expenditure to conduct a multi-day study with multiple sets of equipment.

\section{The Tests:}

Five tests were performed in order to collect data. The subjects were split into the five test groups of 4 subjects each using the same random number generator used previously. Test one acted as the control group, where tests two through five were conducted as experimental runs. Before each subject was tested, the following was read to them :

\footnotetext{
"Thank you for volunteering for this experiment. The purpose of this experiment is to determine the effectiveness of using a PDA while attempting to perform a foreign task while out in the field.

In the next few minutes you will be asked to perform a task. I will time you and record the number of mistakes that you make along the way. Bear in mind that we are trying to measure the effectiveness of the device and not of your performance.

Your task will be to assemble a flashing LED from a series of electrical components."
}

The statement was the same for each of the subjects. The purpose of each test is as follows:

Test One used the included instructions and schematics that came with the transistor board. The times and number of mistakes of each subject were recorded and their mean used as the population mean in computing the $t$ scores of the following 4 tests and testing of the alternative hypothesis in each test..

Test two studied the use of only instructional video. Each subject was permitted to watch a twelve-minute video that showed step by step the actual assembly of the board (this time is not reflected in their time scores). After they finished the video, they were not permitted to review it during assembly or to use the instruction or schematics. The Null (Ho) and alternative (Ha) are as follows:

$\mathrm{Ho}_{\mathrm{t}}$ : There will be no change in the amount of time taken $\left(\mu_{1}=\mu_{2}\right)$

Hat: It will take more time $\left(\mu_{1}<\mu_{2}\right)$

$\mathrm{Ho}_{\mathrm{m}}$ : There will be no change in the number of mistakes $\left(\mu_{1}=\mu_{2}\right)$

$\mathrm{Ha}_{\mathrm{m}}$ : There will be more mistakes $\left(\mu_{1}<\mu_{2}\right)$ 
Test three studied the use of a prior video clip, but unlike test two, allowed for the use of the instructions and schematics during assembly. It did not allow for review of the video during the procedure.

$\mathrm{Ho}_{\mathrm{t}}$ : There will be no change in the amount of time taken $\left(\mu_{1}=\mu_{2}\right)$

Hat: It will take less time $\left(\mu_{1}>\mu_{2}\right)$

$\mathrm{Ho}_{\mathrm{m}}$ : There will be no change in the number of mistakes $\left(\mu_{1}=\mu_{2}\right)$

$\mathrm{Ha}_{\mathrm{m}}$ : There will be fewer mistakes $\left(\mu_{1}>\mu_{2}\right)$

Test four used a Compac Ipac handheld computer to make available the video clip during the assembly of the board. No access to the instructions or schematics was permitted. This test was the first primary test for the study.

$\mathrm{Ho}_{\mathrm{t}}$ : There will be no change in the amount of time taken $\left(\mu_{1}=\mu_{2}\right)$

Hat: It will take less time $\left(\mu_{1}>\mu_{2}\right)$

$\mathrm{Ho}_{\mathrm{m}}$ : There will be no change in the number of mistakes $\left(\mu_{1}=\mu_{2}\right)$

$\mathrm{Ha}_{\mathrm{m}}$ : There will be fewer mistakes $\left(\mu_{1}>\mu_{2}\right)$

Test Five used the same PDA to make the video available, but also allowed for the use of the instructions and schematics. This test was the second primary test for the study.

Hot $_{t}$ : There will be no change in the amount of time taken $\left(\mu_{1}=\mu_{2}\right)$

$\mathrm{Ha}_{\mathrm{t}}$ : It will take less time $\left(\mu_{1}>\mu_{2}\right)$

$\mathrm{Ho}_{\mathrm{m}}$ : There will be no change in the number of mistakes $\left(\mu_{1}=\mu_{2}\right)$

$\mathrm{Ha}_{\mathrm{m}}$ : There will be fewer mistakes $\left(\mu_{1}>\mu_{2}\right)$

Set Up:

The task was performed using a Radio Shack "Electronic Learning Lab" which included an electronic breadboard and loose electronic components which could be placed on the breadboard. The kit also included an printed instructional manual with step-by-step instructions and a schematic diagram. The pages concerning the assembly of the LED were reproduced and provided to those subjects that required them based on their test group assignment.

The authors prepared the video portion of the experiment one day prior to the experiment. The authors used completed the task while taping it with a Canon Elura MiniDV video camera. One of the authors provided an instructional voice-over as he performed the task on camera. The camera remained zoomed into the breadboard at all times and never showed the person performing the task or the voice-over. Only his hands appeared on the tape.

The video tape was then digitized using Vegas Video 2.0 and converted to Window Media 8 at $320 \times 240$ at a data-rate of 100 kilobits per second. A second version of the video was compressed at size of 240x320 (letterboxed) to be shown on the PDA. This was necessary because PocketPC PDA's have a screen which is longer than wide. Thus the size had to be altered to account for differences in aspect ratio. Group two watched the $320 \times 240$ video on a laptop computer. Groups four and five had access to the $240 \times 320$ video from their PocketPC PDA.

"Proceedings of the 2002 American Society for Engineering Education Annual Conference \& Exposition Copyright (C) 2002, American Society for Engineering Education" 
To deliver the video to the user's PDA wirelessly the authors loaded the WMV files onto a laptop running Microsoft Internet Information Server 5.0. An ASP that linked to the WMV file was placed on the same server. During the test, the subject's PDA requested the ASP page from the laptop over an IEEE 802.11b wireless LAN connection.

\section{Results}

In order to compare each of the four experimental tests with the control tests, t-scores were computed for each experimental run using the mean from the control group as the value for the population mean. These scores were then converted to $p$-values and compared to $\alpha$ (Alpha) $=0.10, \alpha=0.05$ and $\alpha=0.01$ in order to test their level of significance. Tests that proved significant at $\alpha=0.10$ were considered a marginal success, tests at $\alpha=0.05$ were considered highly probable successes, and tests that proved significant at $\alpha=0.01$ were considered a certain success. The data for each test is available in Appendix A.

\section{Control (Test 1):}

As test one served as the control group, there are no null or alternate hypothesis. The results in the table below were used to calculate the t-scores and p-values of the next four tests in order to test their Null and alternate hypothesis.

\section{Prior Video Clip; No printed instructions allowed(Test 2):}

Test two was the only test that contained an outlier (subject three). All calculations are corrected to reflect this, with $\mathrm{N}$ being reduced from four to three, and subject three's scores have not been used. It was the belief before the test that it would take longer to complete the task if the subjects if the had to complete the task entirely from memory after watching a video on how to do it. However, there was no evidence to support this. The mean time was actually less than the control. As our alternate hypothesis was one sided, testing only for an increase in time, our conclusion is that watching a video prior to the test, and having no other support, offers no evidence of an increased time-span to completion. The mean number of mistakes in test two proved to be higher than the control, but not by enough to prove significant at our minimum level of alpha $=0.1$. As a result, both null hypothesis were retained.

\section{Prior Video Clip; Printed Instructions allowed (Test 3):}

Test three was a success. The theory before running the test was that having seen an example of the procedure before hand, and then having access to the instruction, each subjects speed would increase over the control. This proved true, yielding a mean time that was less than half of the control mean, and generating a p-value of less than 0.0002 . As a result, the null hypothesis was rejected and the alternate hypothesis, which stated that the test mean would be less than the control, was accepted. On the other hand, the mean number of mistakes (0.5) did not prove low enough to reject the null hypothesis. In the measure of mistakes, the null hypothesis was retained.

\section{PDA Video Clip; No instructions allowed (Test 4):}

Test four was the first of the two primary tests. It was the author's belief that having access to the PDA during the run would greatly increase each subject's speed. 
This was due to the belief that having access to a visual step-by-step guide would help them both in accuracy and in time. The data, however, does not support all of this. While the number of mistakes proved to be significantly lower (at the alpha $=0.1$ level), the mean time was greater than the control, making the alternate hypothesis (which stated it would be lower) impossible to accept.

\section{PDA Video Clip; Instructions Allowed (Test 5):}

Test 5 was the second of the two primary tests. The author's believed that this test would prove the most successful, reducing both the number of mistakes and the time needed to complete the task. The data conclusively supports that it took less time, with it being significant at the alpha $=0.01$ level, our most precise test. However, test five's mean number of mistakes was equal to that of the control. As a result, only the alternative hypothesis for the time taken was accepted.

\section{Conclusion}

Based upon the results of this study, and specifically upon the results of the two primary tests, it is the conclusion of the authors that a PDA, when used properly, can greatly facilitate a technical task. Based solely on this study its proper use would be as an additional tool, to be used alongside printed material. Used only on its own may serve some users, but not the majority. It is the authors belief that the video that the PDA makes available serves as a clarifying agent in cases where the printed instructions are ambiguous (this also seemed to be the case in test three). However, when used solely on its own, a PDA either does not provide enough information, or does not provide in a method that conforms to the user's learning style.

This study should serve as a stepping-stone into a more thorough review of PDA use in instruction. The next study should test the same areas, as the non-PDA tests offered insight into how the subjects best performed. In addition, a posttest interview should be conducted in order to learn what difficulties each subject had in completing the task. The number of subjects should be increased by at least a factor of 5 . With a sample of at least 20 , the statistical analysis of the results will become much more precise. Further exploration of this subjects also needs to consider the use of on-site training PDA's for non-procedural tasks and in situations where the subjects have a some pre-task knowledge.

As a preliminary study, this experiment has shown that PDA's are a useful tool in completing complex tasks that are new to the user. 


\section{Appendix A}

Time to Complete Task

\section{Time to complete task (by group)}

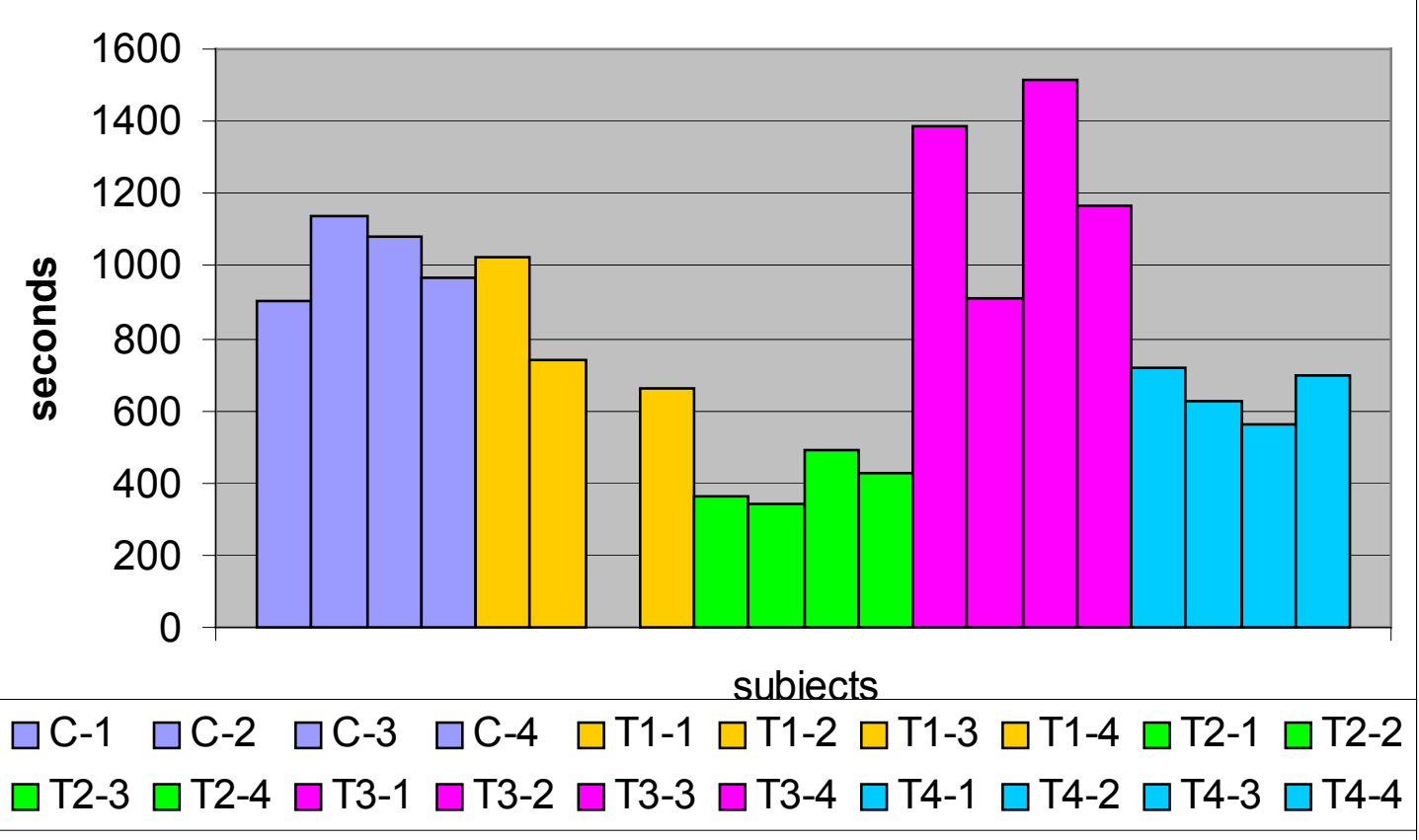

Raw Data and Calculations

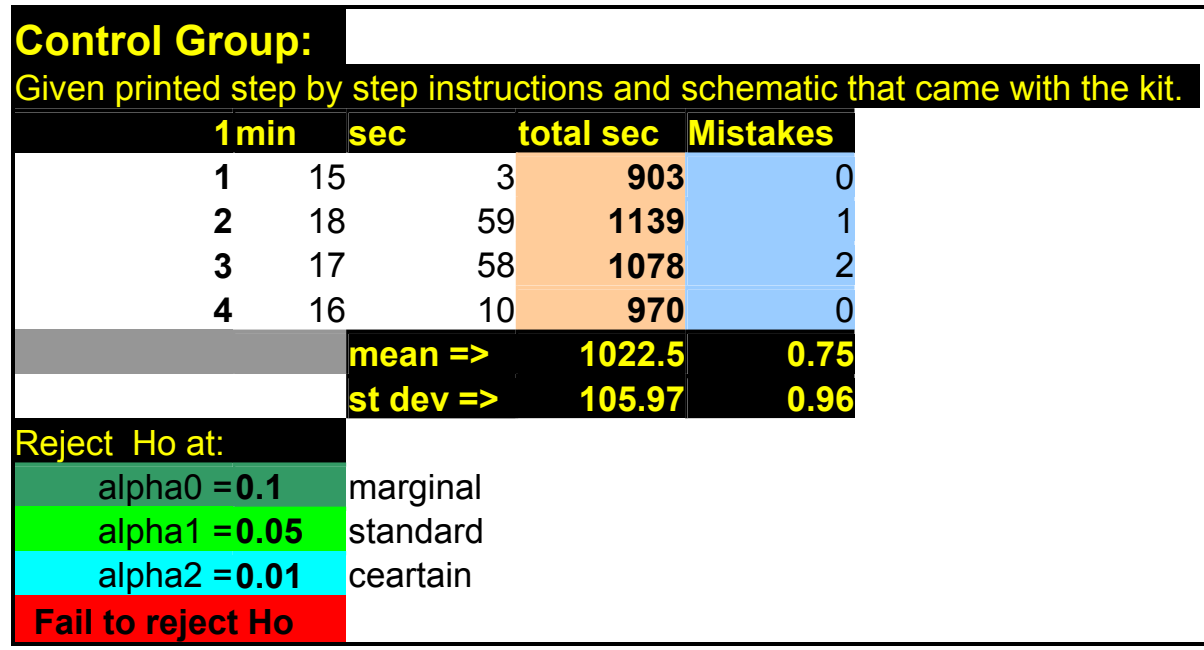



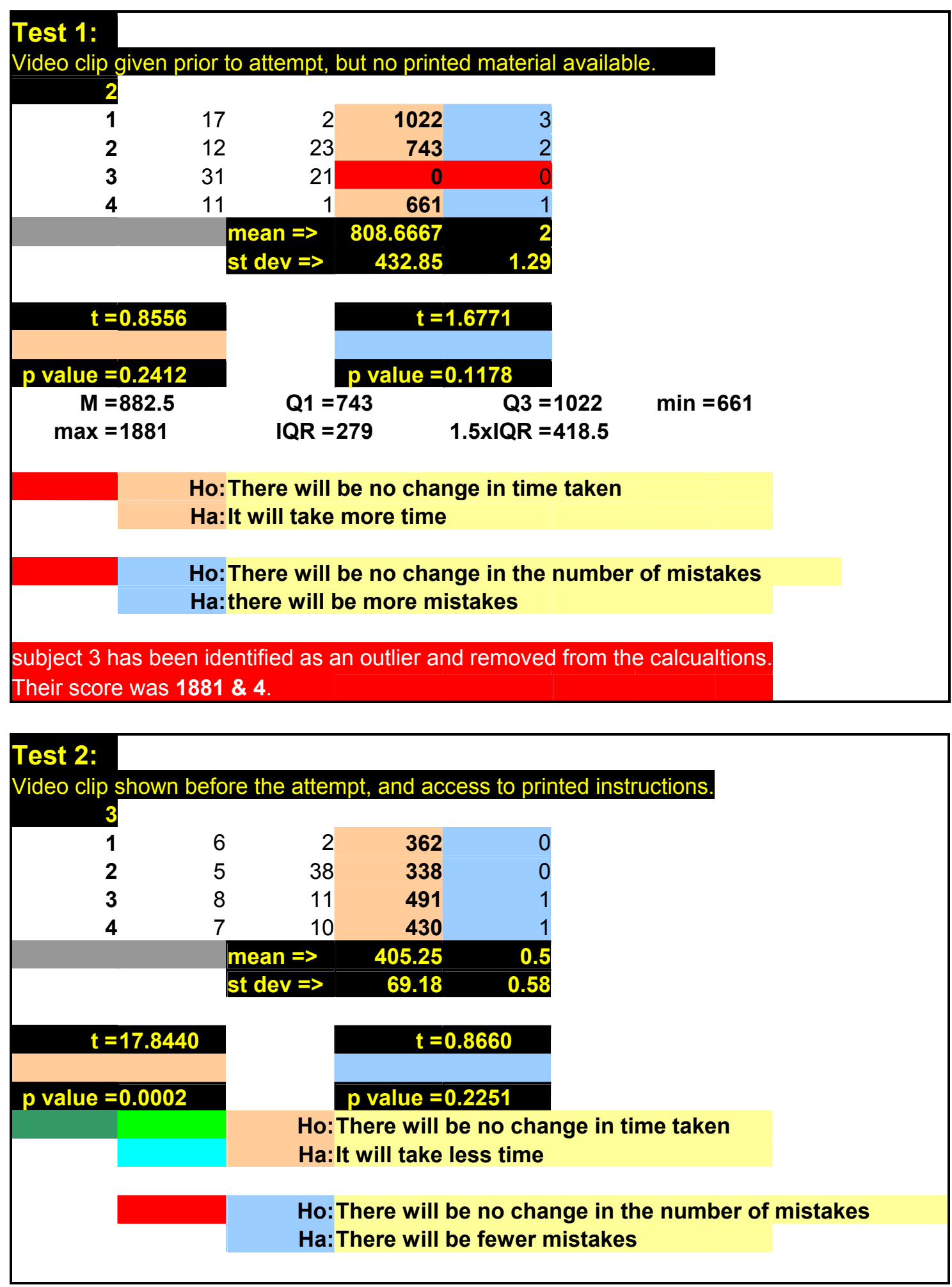

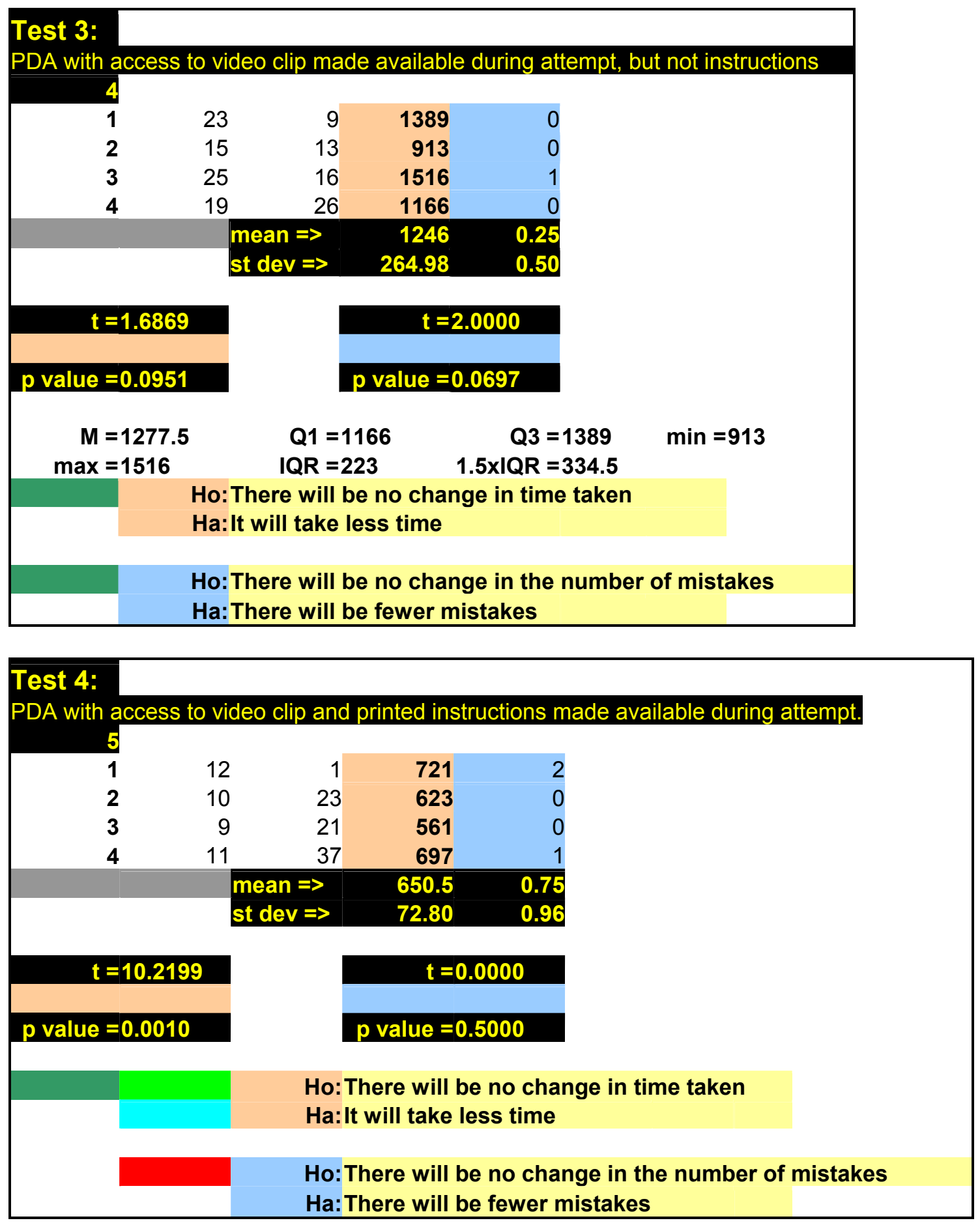

\section{References}

[1] Wireless training or m-learning is here; first movers in the pool. Lifelong Learning Market Report (Vol 5. Issue 22, pp. 3-5). Nov 29,2000. 
[2] Morales, C. (2001). A method of delivering dynamic hyperlinked streaming instructional content over a wireless environment through the use of active server pages and xml technology. 2001 Annual Conference Proceedings . American Society for Engineering Education, Albuquerque, NM.

[3] Shotsberger, P., Vetter, R. (2000). The handheld web: how mobile wireless technologies will change web-based instruction and training. Educational Technology. V40, n5, pp.49-52.

\section{CARLOS MORALES}

Carlos R. Morales is an assistant professor of computer graphics in the Department of Computer Graphics Technology at Purdue University. He holds a BA in Telecommunications and an MS Ed. in Curriculum and Instruction. Prior to working at Purdue University, Carlos worked as a Technical Director. Some of his clients have included Microsoft, Chicago Bulls Organization, First Alert and Brach's Candies. His research interest includes distance learning, animation, and multimedia development.

JASON P. SOLE

Jason P. Sole is a graduate student and teaching/research assistant in the Department of Computer Graphics Technology at Purdue University. He holds a B.S. in Technology. He can be reached at sole@purdue.edu. 